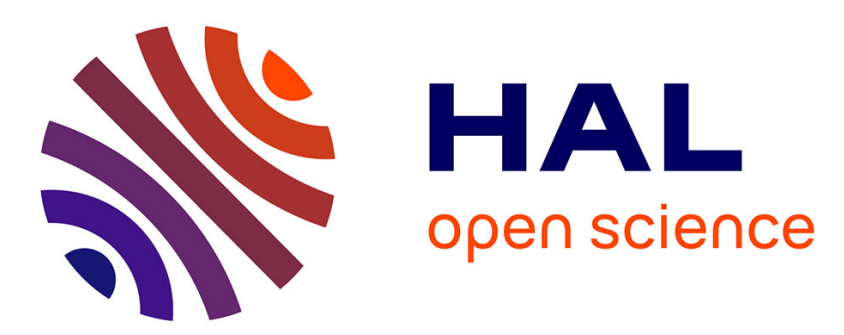

\title{
RECHERCHES SUR LA PASTEURISATION BASSE DU LAIT. UNE NOUVELLE RÉACTION POUR CONTROLER LA TEMPÉRATURE DE CHAUFFAGE (Suite)
}

S. Orla-Jensen

\section{To cite this version:}

S. Orla-Jensen. RECHERCHES SUR LA PASTEURISATION BASSE DU LAIT. UNE NOUVELLE RÉACTION POUR CONTROLER LA TEMPÉRATURE DE CHAUFFAGE (Suite). Le Lait, 1929, 9 (87), pp.724-740. hal-00894959

\section{HAL Id: hal-00894959 https://hal.science/hal-00894959}

Submitted on 1 Jan 1929

HAL is a multi-disciplinary open access archive for the deposit and dissemination of scientific research documents, whether they are published or not. The documents may come from teaching and research institutions in France or abroad, or from public or private research centers.
L'archive ouverte pluridisciplinaire HAL, est destinée au dépôt et à la diffusion de documents scientifiques de niveau recherche, publiés ou non, émanant des établissements d'enseignement et de recherche français ou étrangers, des laboratoires publics ou privés. 


\title{
RECHERCHES SUR LA PASTEURISATION BASSE DU LAIT. UNE NOUVELLE RÉACTION POUR CONTROLER LA TEMPÉRATURE DE CHAUFFAGE
}

\author{
par le Professeur ORLA-JENSEN
}

(Suite)

Si nous faisons abstraction provisoirement du lait des vaches de Jersey, nous voyons que A est constamment du bon côté de 1 à l'exception du lait cru de la vache à courtes cornes 33 , où $\mathrm{A}$ n'est que 0,8 . Ce lait se montra toutefois à l'épreuve à l'alizarol nettement alcalin et ne peut donc pas être considéré comme normal. Le résultat pour le lait des vaches de Jersey isolées est moins favorable. Déjà en ce qui concerne le lait mélangé notre réaction était quelque peu affaiblie, et pour les vaches isolées, nous rencontrons deux cas (Nos 37 et 40) où A pour le lait soumis à la pasteurisation basse est très au-dessus de 1 .

Dans les cas douteux, on peut toutefois utiliser C, qui est le rapport entre la crème dans le lait étendu et la quantité de matière grasse, et qui n'est pas moins caractéristique que le rapport $A$, mais qui est plus difficile à établir, car il exige une détermination de matière grasse dans le lait (1). Le tableau XIV montre les variations et la valeur moyenne du rapport $\mathrm{C}$ dans le lait traité préliminairement. Le lait de Jersey est à part. Les chiffres entre parenthèses ne se sont présentés qu'une fois.

TABLEAU XIV

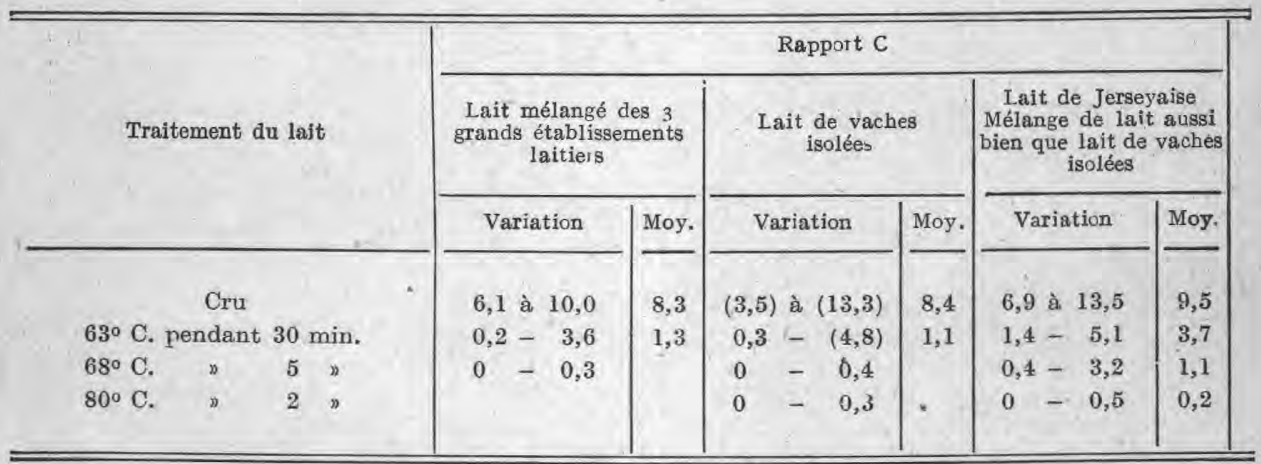

Du tableau XIV, il ressort nettement que le lait de Jersey par rapport à son contenu de matière grasse laisse généralement monter sa crème plus vite (tous nos chiffres se réfèrent à la montée de la crème a près 2 heures) que les autres laits, et que ceci se fait surtout sentir quand le lait a été

(1) C doit toujours être utilisé comme aide, quand les quantités de crème sont très petites car, dans ce cas, le calcul de A est très incertain, en ce sens qu'un seul 1/2 degré Höyberg est décisif, et il peut arriver que A monte de nouveau au-dessus de 1 quand on a chauffé le lait si fortement que la crème ne monte presque pas. 
soumis à la pasteurisation basse. On ne peut pas mettre en doute que ceci tient à ce que les globules de matière grasse sont relativement plus gros dans le lait de Jersey. Le rapport $\mathrm{C}$ peut aussi beaucoup varier, mais il est remarquable que lors de nos recherches $A$ et $C$ n'ont jamais été en même temps anormaux. Dans les laits de Jersey 37,39 et 40 qui, soumis à la pasteurisation lente, ont $\mathrm{A}=2,0,1,0$ et 1,6 , le $\mathrm{C}$ correspondant est $3,3,5,0$ et 5,1 , soit au-dessous de 6,9 , qui est le chiffre le plus bas que nous ayons trouvé pour $\mathrm{C}$ dans le lait de Jersey cru. Nous avons une fois trou vé du lait d'une vache de la race laitière da noice rouge qui, soumis à la pasteurisation lente, avait $\mathrm{A}=1,2$. $\mathrm{C}$ était à cette occasion 2,0 , soit très au-dessous du chiffre le plus bas trouvé par nous dans le lait cru. Le lait cru déjà cité (courtes cornes 33 ) avec $\mathrm{A}=0,8$ a $\mathrm{C}=5,1$, ce qui prouve qu'il est tout de même eru. Si l'on rencontre dans la pratique un lait qui a été soumis à la pasteurisation basse, mais qui a néanmoins $A>1$, il ne faut pas le considérer comme insuffisamment chauffé, à moins que $\mathrm{C}$ ne soit aussi élevé (pour le lait mélangé sans lait de Jersey au-dessus de 3 ).

TABLEAU XV

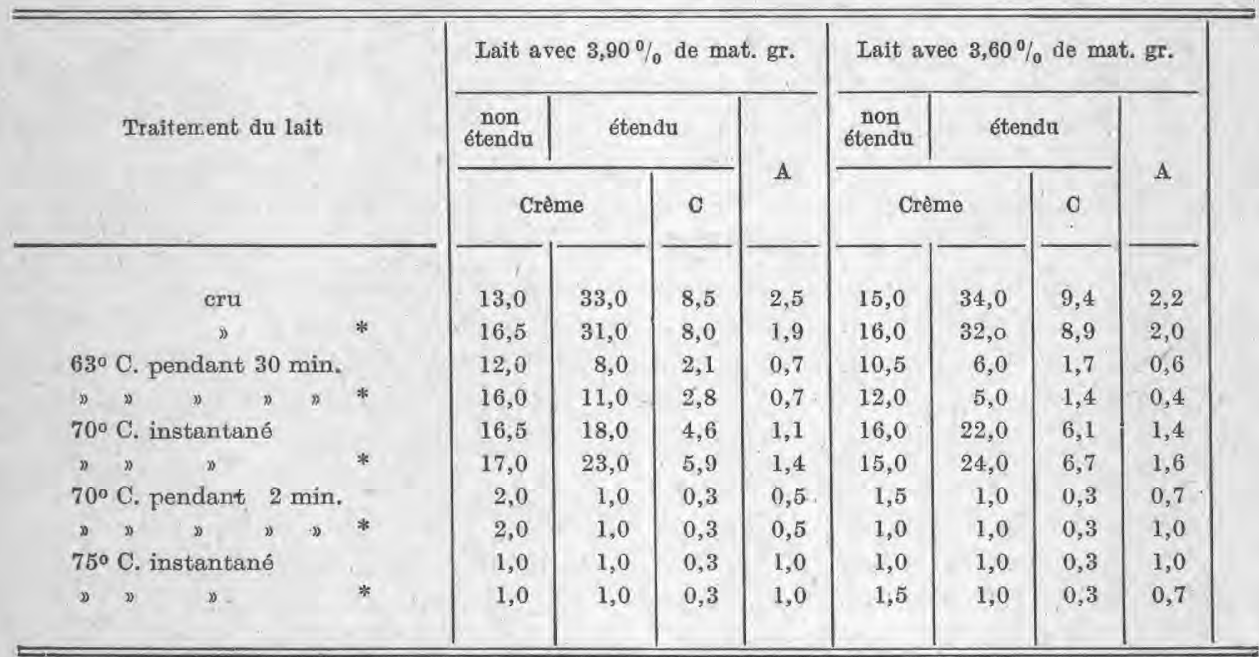

Il ressort du tableau XV qu'à une température élevée comme celle de $70^{\circ} \mathrm{C}$. un petit changement dans le temps de chauffage joue un très grand rôle dans la faculté de montée de la crème du lait. Tandis que cette faculté est à peine influencée par un chauffage instantané à $70^{\circ} \mathrm{C}$., ce qui fait aussi qu'ici A est plus grand que 1 , elle est presque entièrement détruite par 2 minutes de chauffage à $70^{\circ} \mathrm{C}$. A $75^{\circ} \mathrm{C}$, le chauffage instantané est đéjà suffisant pour détruire la faculté de montée de la crème, et c'est pourquoi le lait traité par le procédé Stassano ne produit généralement pas de montée de crème en 2 heures. 
Le traitement du lait qui s'est montré nézessaire lors de la réaction de la montée de la crème pour éviter une confusion entre le lait cru d'écrémage paresseux dû au froid et le lait ayant subi la pasteurisation basse, a toujours été exécuté dans une petite fiole d'Erlenmeyer dans un bain-marie à $50^{\circ} \mathrm{C}$. Aussitôt que le lait a été à la température du bain-marie pendant 5 minutes, on en enlève 10 et 5 cc. a vec une pipette pour les mettre dans les butyromètres, et après que la dernière partie a été mélangée avec soin avec $5 \mathrm{cc}$. d'eau, les 2 parties sont remises dans l'eau courante en vue de la montée de la crème. Comme on pourrait trouver plus facile d'exécuter le traitement préliminaire dans les butyromètres et de mélanger auparavant le lait avec l'eau, nous avons aussi essayé cette méthode. On ne peut toutefois pas l'employer, ear si on évite au lait chauffé à $50^{\circ} \mathrm{C}$. le refroidissement et le travail mécanique (enlèvement à la pipette), avant qu'il ne soit placé, la montée de la crème est aussi, dans le lait étendu ayant été soumis à la pasteurisation lente, si bonne que le rapport A se tient au-dessus de 1 . Dans les tableaux XVI et XVII, le lait soumis au traitement préliminaire dans les butyromètres est marqué **.

Lors de l'essai, dont les résultats sont rassemblés dans le tableau XVI, nous avons mesuré la couche de crème après 1 heure $1 / 2$ puis après les 2 heures habituelles, et on voit qu'après le traitement ** toute la crème est pratiquement déjà montée après 1 heure $1 / 2$, et que par le traitement ** on obtient constamment plus de crème que par le traitement *. Par le traitement ** on obtient encore en 2 heures une montée de crème importante, même dans le lait qui a été chauffé 15 minutes à $65^{\circ} \mathrm{C}$. ou 5 minutes à $67^{\circ} \mathrm{C}$. Si on emploie les 2 méthodes de traitement sur le même lait, on peut non seulement discerner la température inférieure à laquelle il a été chauffé, mais aussi la température la plus élevée à laquelle il a été porté, et les 2 déterminations sont également nécessaires, quand il s'agit de lait soumis à la pasteurisation basse, qui doit être non seulement exempt, ou en tout cas pauvre en bactéries dangereuses, mais qui ne doit pas non plus avoir subi de modifications chimiques marquées.

Ce qu'on peut obtenir par la réaction de la montée de la crème ressort au mieux du tableau XVII, où (comme dans le tableau VII) sont rassemblés les résultats de recherches sur toutes les qualités de lait que les trois grands établissements laitiers de Copenhague vendent. Si nous ne tenons pas compte du lait de Jersey, A est d'abord au-dessous de 1 pour toutes les autres sortes de lait quand le lait a été chauffé $30 \mathrm{mi}$ nutes à $62^{\circ} \mathrm{C}$, Ceci se réfère au traitement *. Pour le traitement **, A est d'abord généralement au-dessous de 1 , quand il a été chauffé 15 minutes à $65^{\circ} \mathrm{C}$. Le rapport $\mathrm{C}$ ne donne pas moins de renseignements précis que le rapport A. Dans tous les cas, il est facile de discerner si le fait a été chauffé 5 minutes à $68^{\circ} \mathrm{C}$, car la faculté de montée de la crème de même que parle traitement ** a beaucoup souffert. 
TABLEAU XVI

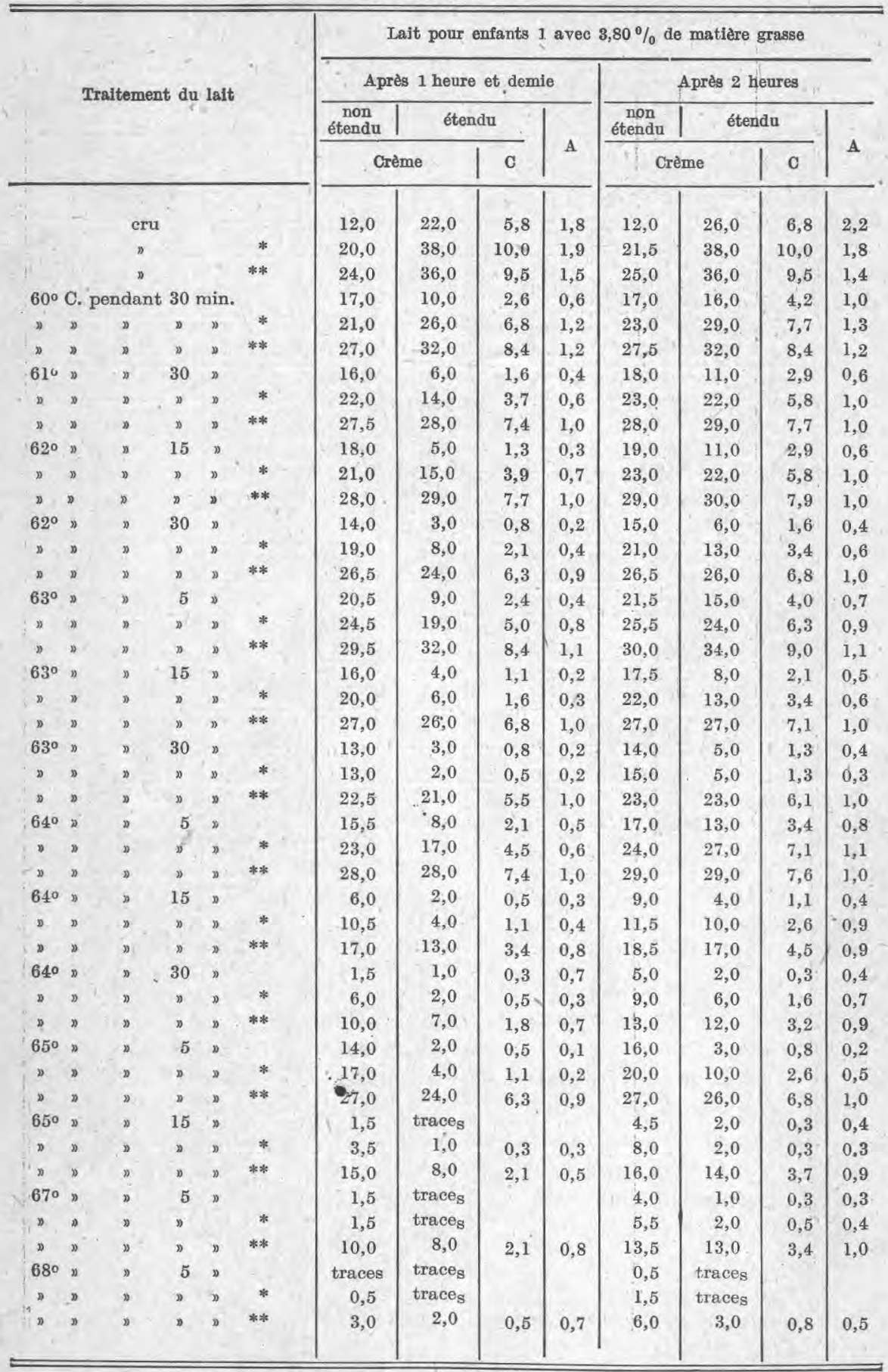




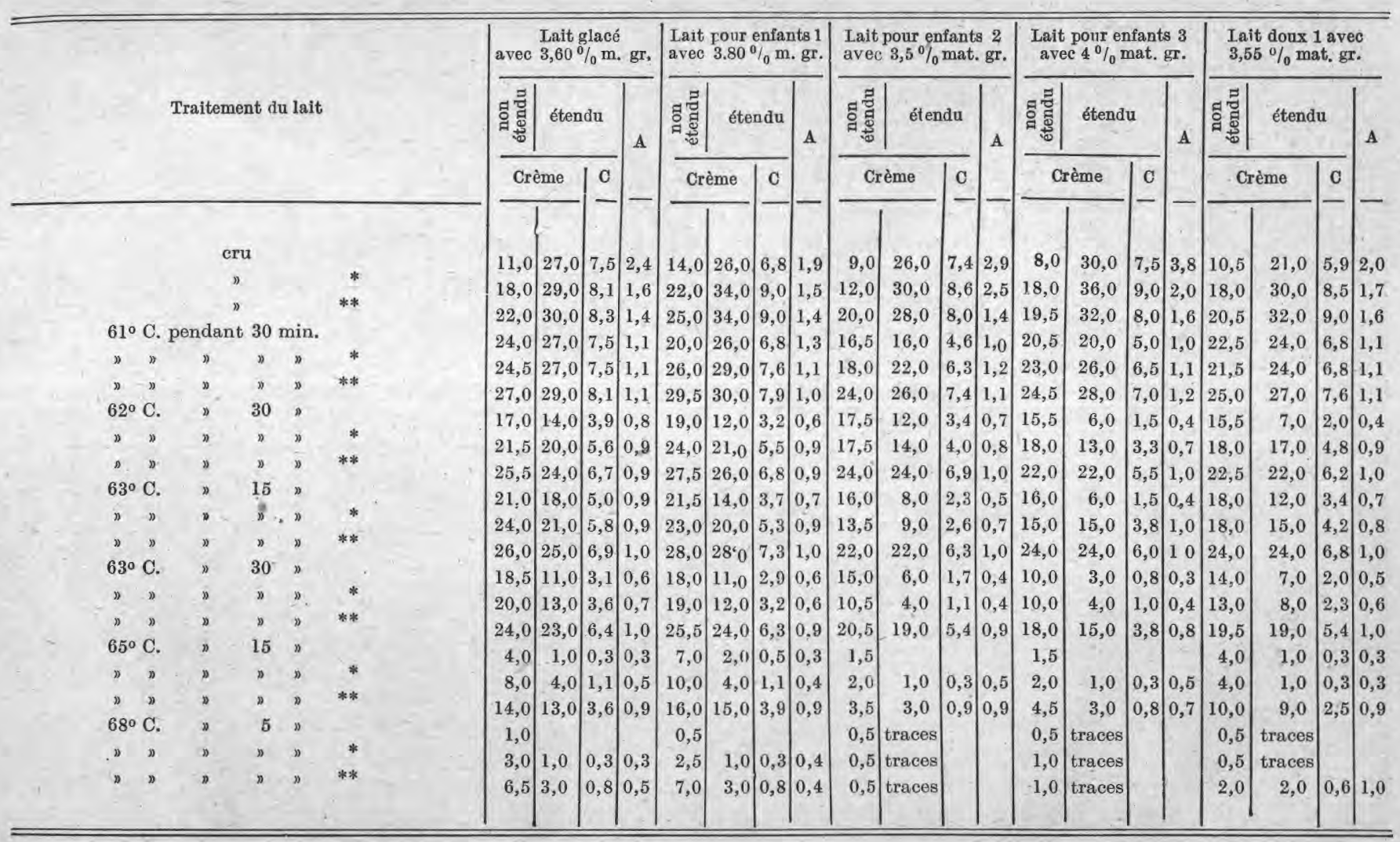


Les tableaux XVI et XVII confirment qu'on obtient la plus grande montée de crème dans le lait non étendu d'eau quand il a été chauffé 30 minutes à $61^{\circ} \mathrm{C}$. Les courbes suivantes indiquent graphiquement comment la montée de la crème du lait est influencée par l'addition d'eau et le chauffage (respectivement le traitement préliminaire).

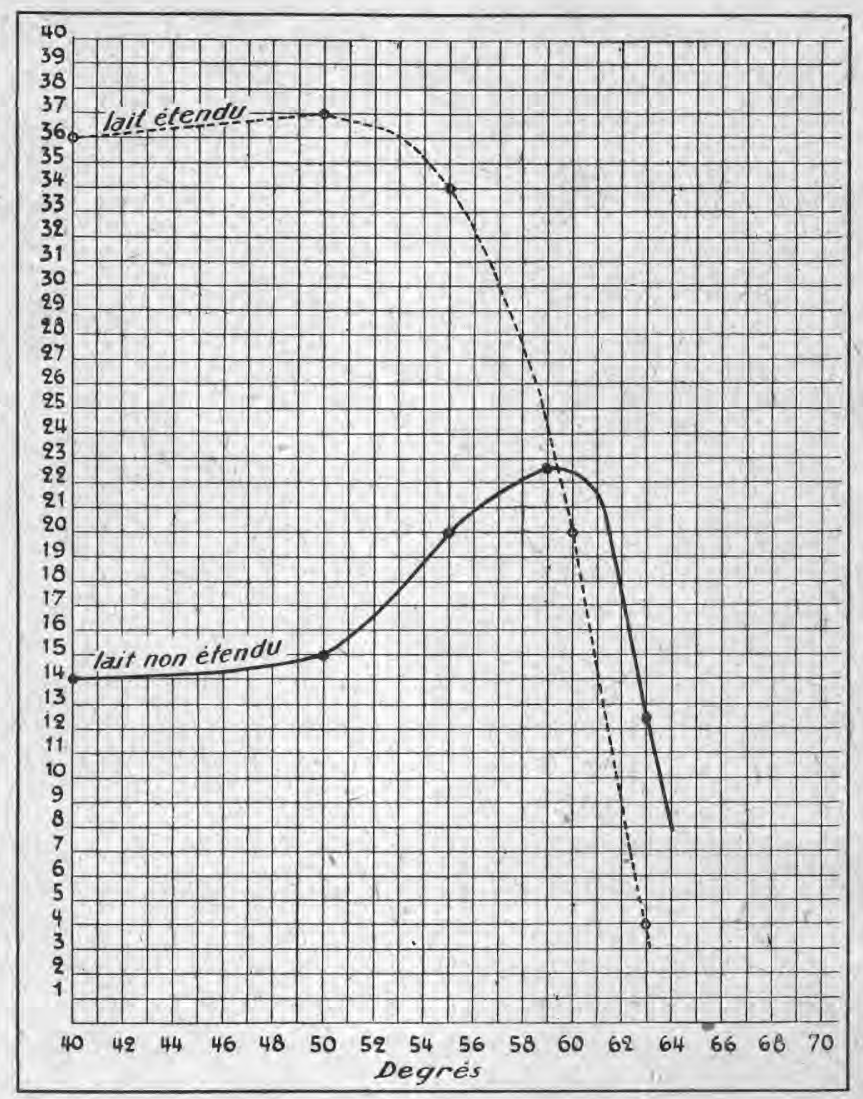

Dans ce qui précède, il n'est pas tenu compte du lait à écrémage paresseux alors qu'il vient d'être tiré; c'est-à-dire du lait qui déjà à l'état tout à fait frais laisse difficilement monter sa crème, et qui par suite ne laisse pas monter de quantité appréciable de crème en 2 heures même après a voir subi le traitement employé par nous pour la suppression de l'écrémage paresseux dû au froid. Jugé uniquement d'après notre réaction de montée de crème, un tel lait sera considéré comme pasteurisé, même s'il est cru.

Lors des recherches sur les laits de vaches isolées, nous avons rencontré quelquefois du lait présentant; alors qu'il vient d'être tiré, les caractéristiques du lait d'écrémage paresseux. Généralement, il s'agissait 
d'un lait extraordinairemer.t gras pour la race en question (par exemple lait d'une vache hollandaise avec $6,10 \%$ de matière grasse), et il s'est toujours trouvé que ce lait provenait d'une vieille laitière, dans les tous derniers jours de la lactation. Une seule fois, nous avons pourtant rencontré un lait d'écrémage paresseux dû à la vache avec une faible teneur en matière grasse $(2,12 \%)$; mais il provenait sûrement d'une vache à pis malade, car il était à la fois alcalin (acidité Soxhlet-Henkel 5,5) et salé (teneur en chlore : $1,38 \%$ contre $0,8 \%$ dans le lait normal).

Mélangé à d'autres laits, le lait de cette sorte ne se fait pas remarquer pour la montée de la crème, et nous pensons que le fait que notre réaction ne peut pas être utilisée en ce qui concerne le lait de vaches isolées présentant de tels écarts n'a pas d'importance pratique, étant donné qu'on ne mettra jamais en vente le lait d'une vieille laitière isolée ou d'une seule vache à pis malade. Dans les villes, il s'agit toujours de lait mélangé, et, dans ce cas, le rapport A complété au besoin, comme nous l'avons $\mathrm{vu}$, par le rapport $\mathrm{C}$, donnera des points de repère certains pour savoir jusqu'à quelle température il a été chauffé. Notre réactioñ ne convient pas aussi bien pour le lait de Jersey, et ceci non seulement parce que, dans ce cas, le rapport A, comme nous l'avons dit, est toujours tout près $\mathrm{de} 1$, mais aussi paroe qu'on peut rencontrer du lait de Jersey mélangé qui, à l'état cru, ne montre pas de ligne de séparation nette entre la crème et le lait.

A cet égard, il faut encore noter une source d'erreurs, qui estla division extrême (homogénéisation) que les globules de matière grasse du lait peuvent subir sous l'influence d'un traitement mécanique, et qui peut agir très fortement sur la montée de la crème.

Si le traitement mécanique du lait a lieu à basse température, même à $20-30^{\circ} \mathrm{C}$., ces effets peuvent généralement être anníhilés complètement ou partiellement par le traitement préliminaire employé par nous (5 minutes de chauffage à $50^{\circ} \mathrm{C}$.), et c'est pourquoi ces effets ont plutôt été la cristallisation favorisée dans les globules de matière grasse sousrefroidis que la subdivision de ceux-ci. Si par contre le lait a $40^{\circ} \mathrm{C}$, ou plus, il n'y a pas de cristallisation dans les globules de matière grasse, mais au contraire ceux-ci se cassent en morceaux, ce qu'on peut suivre directement sous le microseope en faisant à de petits intervalles des préparations de lait chaud soumis à un traitement mécanique.

Le tableau XVIII montre comment la pompe centrifuge, le purificateur centrifuge et enfin l'agitation à basse température dont il a été question précédemment, diminuent la faculté de montée de la crème du lait. En ce qui concerne le lait non étendu, cet état ne peut pas être modifié par notre traitement préliminaire, au contraire. Si, par contre, on étend le lait a près ce traitement, il semble presque regagner toute sa faculté de montée de crème originelle. Nous retrouvons exactement les mêmes circonstances extraordinaires que pour le lait doux $3 \mathrm{du}$ tabl 
VII, et nous comprenons pourquoi ce lait est devenu ce qu'il est. Si nous considérons au contraire le lait 3 du tableau XVII, nous voyons qu'en ce qui concerne la faculté de montée de la crème, il n'est pas en arrière du lait des autres établissements laitiers. Ceci tient à l'acquisition d'un purificateur centrifuge plus lent et à d'autres petites mcdifications.

TABLEAU XVIII

\begin{tabular}{|c|c|c|c|}
\hline & & me & \\
\hline Traitement du lait & 형령 & ఫี & A \\
\hline Lait « a "pris avant l'épuration centrifuge & $\begin{array}{l}14,0 \\
13,0\end{array}$ & $\begin{array}{l}25,0 \\
33,0\end{array}$ & $\begin{array}{l}1,8 \\
2,5\end{array}$ \\
\hline Laít " a "pris après l’épuration centrifuge & $\begin{array}{l}9,0 \\
8,0\end{array}$ & $\begin{array}{l}20,0 \\
32,0\end{array}$ & $\begin{array}{l}2,2 \\
4,0\end{array}$ \\
\hline Lait $\star \mathrm{b}$ " pris avant la pompe centrifuge & $\begin{array}{l}15,5 \\
14,0\end{array}$ & $\begin{array}{l}26,0 \\
32,0\end{array}$ & $\begin{array}{l}1,7 \\
2,3\end{array}$ \\
\hline $\begin{array}{l}\text { Lait } \propto \mathrm{b} \| \text { pris après la pompe centrifuge, mais avant l'épu- } \\
\text { ration centrifuge }\end{array}$ & $\begin{array}{l}12,0 \\
11,0\end{array}$ & $\begin{array}{l}26,0 \\
32,0\end{array}$ & $\begin{array}{l}2,2 \\
2,9\end{array}$ \\
\hline Lait \& $b$ " pris après l'épuration centrifuge & $\begin{array}{l}7,0 \\
5,5\end{array}$ & $\begin{array}{l}19,0 \\
29,0\end{array}$ & $\begin{array}{l}2,7 \\
5,3\end{array}$ \\
\hline Lait " $c$ * refroidi à $301 / 2$ C. dans la cuve réfrigérante & $\begin{array}{l}6,5 \\
2,0\end{array}$ & $\begin{array}{l}12,0 \\
28,0\end{array}$ & $\begin{array}{r}1,9 \\
14,0\end{array}$ \\
\hline $\begin{array}{l}\left.\text { Lait \& e } n, 7 \text { heures plus tard ( } 4^{\circ} 1 / 2 \text { C. }\right) \text {, légèrement reınué } \\
\text { entre temps. }\end{array}$ & $\begin{array}{l}4,0 \\
1,0\end{array}$ & $\begin{array}{r}7,0 \\
28,0\end{array}$ & $\begin{array}{r}1,8 \\
28,0\end{array}$ \\
\hline
\end{tabular}

Le tableau XIX donne les résultats d'un essai dans lequel le lait fut fouetté vigoureusement avec un fouet à crème actionné mécaniquement qui remplissait entièrement le récipient dans lequel se trouvait le lait, de façon qu'aucune portion de lait n'évitait le traitement. Comme on le voit, le lait commence déjà à $40^{\circ} \mathrm{C}$. à être homogénéisé a près 2 minutes de fouettage, en ce sens qu'ensuite, a près les 2 heures habituelles de délai, il ne donne que très peu de crème. Après 4 et surtout après 5 minutes de fouettage, la montée de la crème est, même après 20 heures de délai, extrêmement réduite, ce qui laisse supposer une homogénéisation presque complète.

Nous n'avons pas réussi de la même manière à effectuer les essais à $30^{\circ} \mathrm{C}$. et à $20^{\circ} \mathrm{C}$., car la formation du beurre est déjà visible à ces tem- 
pératures après quelques minutes seulement de fouettage, et s'il se forme tout d'abord de petits flocons de beurre dans le lait, il n'est plus possible d'en prélever des échantillons moyens. On voit toutefois que l'homogénéisation avance beaucoup plus lentement à ces températures, puisque le lait, a près 2 minutes de fouettage à $30^{\circ} \mathrm{C}$. et 3 minutes de fouettage à $20^{\circ} \mathrm{C}$., laisse monter encore beaucoup de crème pendant 2 heures.

TABLEAU XIX

\begin{tabular}{|c|c|c|c|c|c|c|c|c|c|c|c|}
\hline \multirow{3}{*}{ foue } & \multirow{3}{*}{\multicolumn{5}{|c|}{$\begin{array}{l}\text { Lalt pour enfants } \\
\text { mat. gr. } 4,05 \% \% \\
\text { tté avec un fouet à crème }\end{array}$}} & \multicolumn{3}{|c|}{ Après repos de 2 heures } & \multicolumn{3}{|c|}{ Après repos de 20 heures } \\
\hline & & & & & & \multicolumn{2}{|c|}{ Crème } & \multirow{2}{*}{$\mathbf{A}$} & \multicolumn{2}{|c|}{ Crème } & \multirow{2}{*}{ A. } \\
\hline & & & & & & non étendu & étendu & & non étendu & étendu & \\
\hline $40^{\circ} \mathrm{C}$ & C. 1 & nda & & uin. & 4 & 9,0 & 38,0 & 4,2 & 16,0 & 32,0 & 2,0 \\
\hline$\cdot$ & 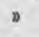 & x & » & o & $*$ & 11,5 & 37,0 & 3,3 & 18,0 & 32,0 & 1,7 \\
\hline $40^{\circ}$ & 》 & $D$ & 1 & b & & pas net & 40,0 & & 25,0 & 44,0 & 1,8 \\
\hline D & v & w & n & D & * & $13, \tilde{5}$ & 40,0 & 2,9 & 26,0 & 40,0 & 1,5 \\
\hline $40^{\circ}$ & $n$ & $n$ & 2 & 》 & & pas net & 2,0 & & 22,0 & 40,0 & 1,8 \\
\hline " & " & » & D & $\infty$ & $*$ & 3,0 & 4,0 & 1,3 & 20,0 & 34,0 & 1,7 \\
\hline $40^{\circ}$ & 》 & 》 & 3 & D & 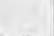 & pas net & 2,0 & & 16,0 & 30,0 & 1,9 \\
\hline x & D & D & D & D & $*$ & 2,0 & 5,0 & 2,5 & 17,5 & 32,0 & 1,9 \\
\hline $40^{\circ}$ & 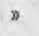 & " & 4 & 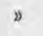 & & traces & traces & 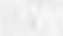 & 5,0 & 12,0 & 2,4 \\
\hline ") & ") & $D$ & " & $"$ & $*$ & 0,5 & traces & & 8,0 & 12,0 & 1,5 \\
\hline $40^{\circ}$ & n & 》 & 5 & $n$ & & traces & 0 & & 2,0 & 3,0 & 1,5 \\
\hline 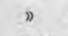 & 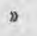 & 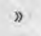 & $n$ & » & $*$ & traces & 0 & & pas net & 6,0 & \\
\hline $30^{\circ}$ & » & ") & 0 & 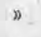 & & 12,0 & 42,0 & 3,5 & 17,0 & 38,0 & 2,2 \\
\hline$n$ & D & D & $n$ & " & $*$ & 16,0 & 36,0 & 2,3 & 19,0 & 34,0 & 1,8 \\
\hline $30^{\circ}$ & 》) & 》) & 1 & " & 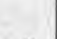 & 11,0 & 36,0 & 3,3 & 17,0 & 34,0 & 2,0 \\
\hline$\Rightarrow$ & $n$ & " & $n$ & ” & $*$ & 14,0 & 30,0 & 2,1 & 17,0 & 30,0 & 1,8 \\
\hline $30^{\circ}$ & ") & 》 & 2 & 》 & $*$ & 11,0 & 20,0 & 1,8 & 13,0 & 22,0 & 1,7 \\
\hline $20^{\circ}$ & D & D & 0 & 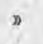 & & 12,0 & 28,0 & 2,3 & & 28,0 & $x^{2}$ \\
\hline$D$ & D & w & " & ") & * & 17,0 & 35,0 & 2,1 & & 32,0 & \\
\hline $20^{\circ}$ & ») & ” & 1 & 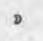 & & 18,0 & 31,0 & 1,7 & 16,0 & 26,0 & 1,6 \\
\hline$D$ & » & 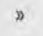 & ") & 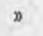 & $*$ & 16,0 & 36,0 & 2,3 & 17,5 & 32,0 & 1,8 \\
\hline $20^{\circ}$ & " & D & 2 & ") & & 14,5 & 28,0 & 1,9 . & 16,0 & 24,0 & 1,5 \\
\hline$"$ & 》 & ” & is & ") & $*$ & 14,0 & 30,0 & 2,2 & 16,0 & 28,0 & 1,8 \\
\hline $20^{\circ}$ & 2 & D & 3 & 》 & 2 & 6,0 & 20,0 & 3,3 & 11,0 & 20,0 & 1,8 \\
\hline 0 & 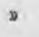 & D & 》 & " & * & 10,0 & 22,0 & 2,2 & 12,0 & 22,0 & 1,8 \\
\hline
\end{tabular}

Pour connaître le pourcentage de matière grasse qui doit être homogénéisé avant que la montée de crème n'en soit gênee, nous avons constitué du lait doux artificiel avec du lait écrémé, de la crème ordinaire (à $40 \%$ de matière grasse) et de la crème homogénéisée (avec $20 \%$ de matière grasse), le tout à l'état cru. En faisant varier la proportion entre les 2 sortes de crème, nous avons obtenu du lait, dans lequel une plus ou moins grande partie de la matière grasse était homogénéisée, et nous a vons trouvéalors que même si $17 \%$ de la matière grasse était homogénéisée, la faculté de montée de la crème n'était pas diminuée visiblement. Si, par 
contre, $30 \%$ de la matière grasse sont homogénéisés, nous tombons à une montée de crème (après 2 heures de délai) aussi petite qu'avec le lait du précédent essai après 2 minutes de fouettage à $40^{\circ} \mathrm{C}$.

Comme il est évident que du lait entièrement ou partiellement homogénéisé réagira lors de notre réaction de montée de crème comme du lait ayant subi la pasteurisation basse, même s'il est cru, il est nécessaire, si on veut baser le contrôle de la pasteurisation basse sur cette réaction, non səulement de déiendre toute addition de lait ou de crème homogénóisés au lait doux ordinaire, ce qui du reste est interdit au Danemark, mais il faut aussi exiger que le lait qui est vendu comme ayant été soumis à la pasteurisation basse n'ait pas subi un traitement mécanique (pompage, nettoyage par purificateur centrifuge, etc...) à des températures supórieures à $20^{\circ} \mathrm{C}$. Une təlle interdiction ne pourra qu'améliorer la qualité du lait. Un examen au mieroscope permettra généralement de désidər si le lait a été traité avec ménagements ou non.

TABLEAU XX

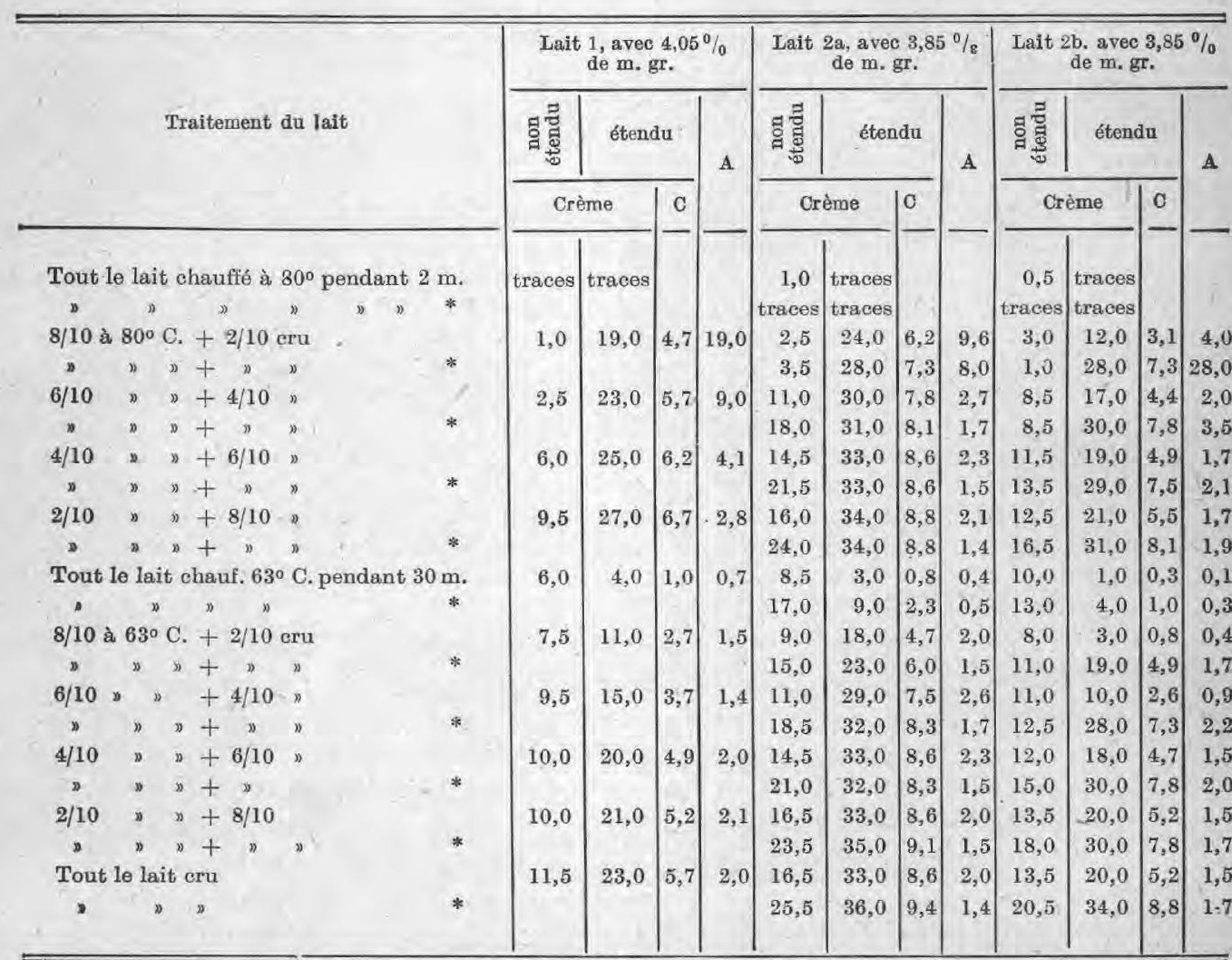


Il s'ensuit naturellement qu'il doit être interdit de mettre en vente un mélange de lait cru et de lait pasteurisé ; du reste, notre réaction montrera de suite un mélange proportionnellement petit de lait cru et de lait pasteurisé, ainsi que les tableaux XX et XXI le montrent.

TABLEAU XXI

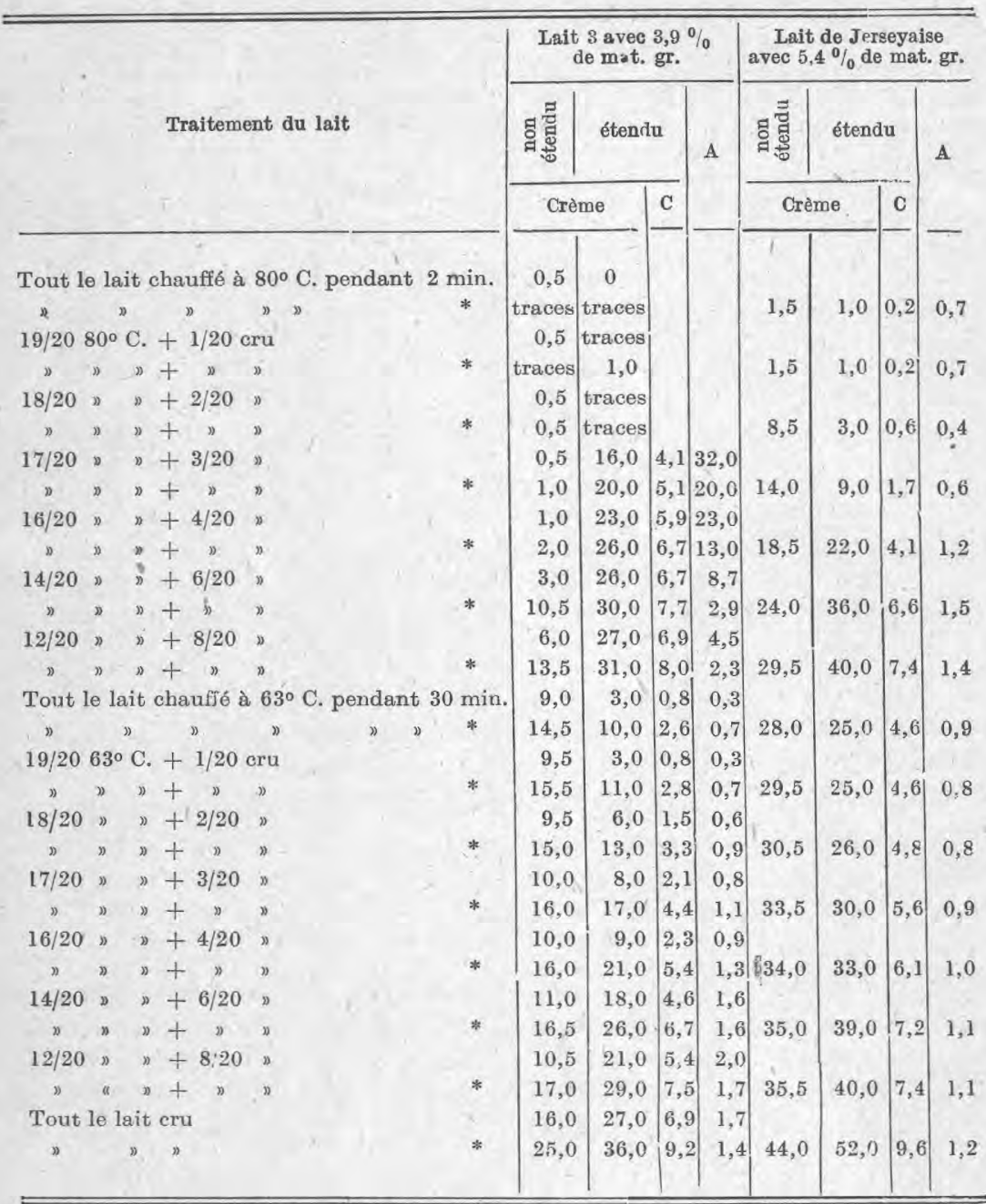

Si on regarde d'abord la deuxième moitié de ces tableaux relative au lait ayant subi la pasteurisation lente, on voit que le rapport A s'élève au-dessus de 1, si on l'additionne seulement de 2/10 (1/5) de lait cru ( $€ n$ tout cas, quand le lait a été soumis au traitement *), Si nous regardons le lait 3 du tableau XXI, A vient déjà au-dessus de 1 si on ajoute seulement 
$3 / 20$ de lait cru. En ce qui concerne le lait de Jersey, il faut bien $4 / 20$ (1/5) de lait cru pour obtenir A au-dessus de 1 , mais ceci est dû à la forte montée de crème de ce lait même à l'état non étendu, car précisément la montée de crème du lait de Jersey s'améliore si seulement on l'additionne de $2 / 20(1 / 10)$ de lait cru. Nous a vons les mêmes limites pour le lait ayant subi la pasteurisation haute (voir la moitié supérieure des tableaux) et A augmente ioi très fortement (à part le lait de Jersey) par de petites additions de lait cru, ce qui, - dans le cas d'un lait ayant subi la pasteurisation haute et qui néanmoins réagit au Storch, - permet de décider si ceci est dû au chauffage insuffisant de tout le lait ou à l'addition d'un peu do lait eru à un lait suffisamment chauffé. L'explication de la forte augmentation de A est que, dans le lait ayant subi la pasteurisation haute, la plus grande partie de l'albumine est.précipitée en fils ou filaments très fortement ramifiés. Dans le lait non étendu la viscosité est trop grande pour que ceux-ci puissent se déplacer sensiblement pendant les deux heures que nous employons pour la montée de la crème. Par contre, dans le lait étendu, ils ont de plus grandes facilités de déplacement, ils sont entraînés par les globules de matière grasse du lait cru qui montent très rapidement, arrêtent eux-mêmes en route une grande partie des globules de matière grasse qui montent lentement et sont en fin de compte si chargés de ballons de matière grasse, que tout l'ensemble est mis en route très rapidement. Si on ajoute à la crème ainsi formée un peu de fuchsine, et si on l'examine au microseope, on voit une image qui rappelle un chêne-marin (fucus vesiculosus), des filaments rouges et ramifiés d'albumine entièrement ornés de globules de matière grasse.

$2 \mathrm{~b}$ est le même lait que $2 \mathrm{a}$; il est seulement resté froid pendant 24 heures pour se rapprocher des conditions de la pratique. Dans ce lait devenu un lait d'écrémage paresseux dû au froid, le traitement préliminaire donne comme d'habitude un très fort résultat, quand le lait est ensuite étendu, tandis que ce traitement ne peut pas au même degré réparer le lait non étendu.

Nous arrivons maintenant au point principal de la réaction de montée de la crème qui est de savoir pourquoi la faculté de montée de la crème du lait est détruite par le chauffage ? A cette question Otto RAHN (1) a répondu apparemment quand il a remarqué que tandis que les globules de matière grasse du lait cru ont tendance à se rassembler, ils restent isolés dans le lait euit, et comme il y a une plus grande légèreté dans un ensemble de globules de matière grasse que dans les petits globules de matière grasse séparés (le phénomène rappelle l'action de la levure dans la fermentation haute et la fermentation basse), on comprend facilement pourquoi la montée de la orème est plus rapide dans le lait cru que dans le lait chauffé.

(1) “Untersuchungen über die Rahmbildung " (Essais sur la formation de la crème). Forschungen, $1921,5,6$ ot 7 . 
Nous avons pu confirmer l'exactitude de cette observation. En gouttes suspendues, on ne voit réellement pas de différence, en ce sens que le champ de vision est rapidement et entièrement couvert de globules de matière grasse, mais, dans une chambre humide de Ranvier, qu'on remplit entièrement de lait (de préférence étendu avec autant d'eau) et qui est placée à peu près verticale, tandis quele microscope est placé à peu près horizontal, on voit que les globules de matière grasse dans leur chemin ascensionnel se rassemblent en grumeaux si le lait est cru, mais se tiennent isolés si le lait a été si fortement chauffé que la faculté de montée de la crème est détruite.

Ceci ne résout cependant pas la question, car, en demière analyøe, il reste à dire, ce qui est spécialement intéressant, la raison pour laquelle les globules de matière grasse se rassemblent en grumeaux dans le lait cru et pas dans le lait chauffé.

RAHN explique cela simplement en disant que ce sont les matières albuminoïdes enveloppant les globules de matière grasse qui ont été dénaturées par le chauffage et qui par conséquent les empêchent d'adhérer ensemble. Cette explication ne peut pas être exacte : cela ressort de ncs essais déjà mentionnés d'addition de lait cru au lait chauffé qui montrent que même si seulement $3 / 20$ de tous les globules de matière grasse n'ont pas été chauffés, nous avons tout de même une montée de crème vigoureuse. L'explication est tout autre : elle ressort des essais suirants faits a vec le lait composé de crème et de lait écrémé où chacunde ces composants avait été chauffé isolément de différentes manières. Pour a voir des essais précis et pour ne pas apporter avec la crème trop des constituants du lait écrémé, il faut employer une crème avec au moins $40 \%$ de matière grasse (1).

Un regard sur le tableau XXII nous persuade de suite que c'est dans le plasma du lait lui-même qu'ont lieu les modifications, qui sont la raison de la destruction de la faculté de montée de la crème quand le lait est chauffé. Dans des essais spéciaux, nous nous sommes ₹ssurés que la montée de la crème ne souffre pas, même si la crème isolée a été chauffée 30 minutes à $80^{\circ} \mathrm{C}$., 5 minutes à $100^{\circ} \mathrm{C}$. ou instantanément à $115^{\circ} \mathrm{C}$. Ce n'est qu'après 5 minutes de chauffage à $115^{\circ} \mathrm{C}$. - chauffage par lequel la caséine commence à brunir - que la montée de la crème est quelque peu affectée. Ceci prouve que les membranes des globules de matière grasse ne peuvent pas être constituées par des albumines coagulables par la chaleur comme par exemple la globuline, mais très probablement d'une mucine comme Storch l'a indiqué (2), ou plutôt de caséine

(1) RAHN a précisément le malheur d'être égaré au lieu d'être guidé, car, dans son seul essai de cette nature, il utílise une crème tellement maigre qu'il doit en employer plus des $3 / 20$, ce qui, quand elle est crue, ainsi que nous l'avons montré, est suffisant pour donner au plasma du lait chauffé la faculté de montée de la crème.

(2) Forsogslaboratoriets 36. Beretning, 1896. (36e rapport du Laboratoire d'Essais agricoles du Danemark, 1896). 
ou d'une substance analogue à la easéine, comme l'indiquent les recherches de Titus, Sommer et Hart (1).

Le tableau montre que le lait composé donne des résultats extraordinairement élevés pour le rapport A, tels qu'ils ne descendent pas au-dessous de 1 , même si le plasma du lait a subi la pasteurisation. Ceci provient de ce que le lait composé à l'état non étendu a une montée de crème tres mauvaise. Comme nous avons donné exactement à ce lait

TABLEAU XXII

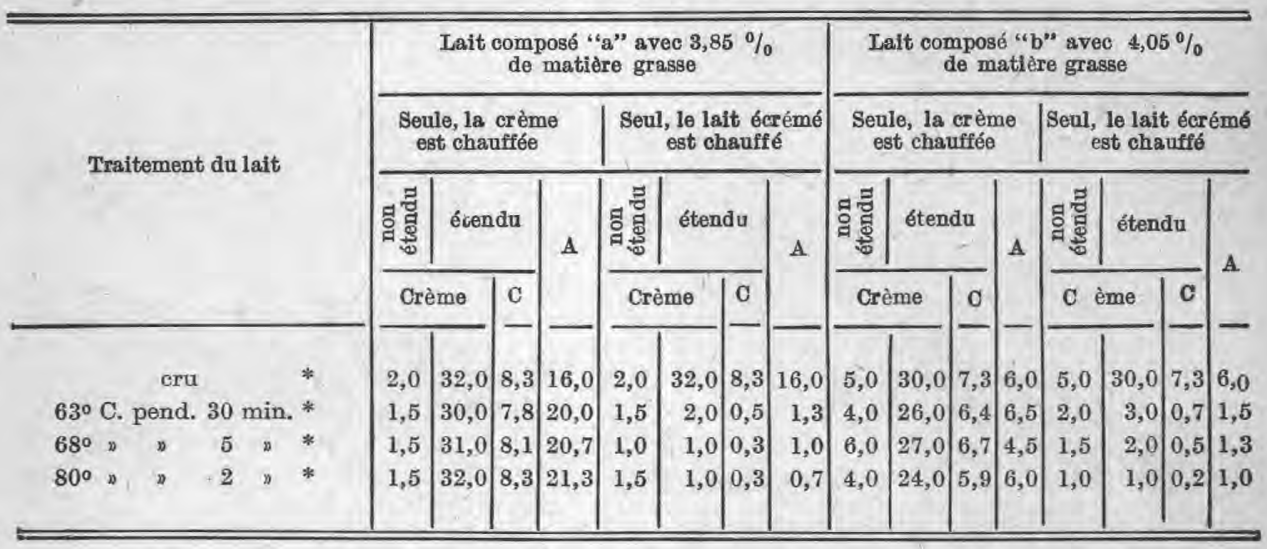

la même teneur en matière grasse qu'au lait dont nous étions partis, une comparaison est possible. La quantité de crème dans le lait "a " original était 16 à l'état non étsndu et 32 à l'état étendu et dans le lait " b " original : 15 à l'état non étendu et 30 à l'état étendu. Le tableau XXII montre les chiffres correspondants après séparation et mélange. Ils sont (grâce à notre traitement préliminaire) très exactement les mêmes dans le lait étendu, mais seulement respectivement 2 et 5 dans le lait non étendu. Ceci détruit une fois pour toutes l'affirmation naïve souvent entendue que, dans un lait préparé en mélangeant du lait écrémé avec de la crème, la crème monte beaucoup plus vite que normalement. C'est précisément le contraire qui se produit, ce qui tient à ce que la crème, lors de la centrifugation (eneore que celle-ci dans les essais en question ait eu lieu à la tempórature de la chambre), subit un tel mauvais traitement qu'elle ne peut plus le surmonter. Nous nous sommes assuré que si nous mélangeons de la crème séparée (dans un vase de séparation à $0^{\circ} \mathrm{C}$.) par montés naturelle avec le lait écrémé correspondant, on obtient un lait doux a vec monté de crème normale, ni plus rapide, ni plus lente, que celle du produit initial. Le fait que la crème est ainsi a bîmée par la centrifugation est un phénomène dont il faut tenir compte si on désire standar-

(1) Nature de la protéine entourant les globules de matière grasse dans le lait (The Journal of Biological Chemistry, 1928, vol. 76, page 237). 
diser le lait à une teneuren matière grasse déterminée. Il ne faut done pas centrifuger tout le lait puismélangerlelait écrémé et la crème en proportions convenables, car on obtient ainsi un lait avec une montée de crème très mauvaise. $\mathrm{Si}$ on veut avoir du lait standardisé avec une bonne montée de crème, il ne faut pas centrifuger plus de lait qu'il n'est nécessaire pour avoir suffisamment de crème ou de lait écrémé dans l'obtention de la proportion voulue de matière grasse. Toutefois, le but de la standardisation du lait destiné à la consommation citadine étant de pouvoir le vendre meilleur marché, il est plussaged'obtenir une teneur en matière grasse un peu en-dessous de la tereur moyenne en matière grasse du lait naturel courant, et c'est pourquoi, pratiquement, il ne sera jamais question d'ajouter de la crème, mais seulement du lait écrémé, ce qui est sans influence sur la faculté de montée de la crème du lait.

Comme lors du chauffage du lait e'est dans le plasma que la faculté de montée de la crème est détruite, rien ne s'oppose à l'emploi de notre réaction sur le lait écrémé, en l'additionnant seulement d'un peu de crème grasse. De ce qui précède, il résulte toutefois que le rapport A ne peut pas être employé dans ce cas, mais seulement le rapport $C$.

A l'inverse de RAHN, la plupart des autres investigateurs ont cherché la eause de la modification que la faculté de montée de la crème subit lors du chauffage du lait, dans des modifications de la viscosité du plasma du Iait; mais, tout d'abord, ces modifications sont si minimes qu'elles sont dans les limites des différences de viscosité qu'on peut trouver dans le lait de vache cru ; elles vont en outre partiellement (par exemple précisement aux environs de $63^{\circ} \mathrm{C}$.) en sens inverse (1), et enfin RAHN a montré que, si on augmente la viscosité du lait par addition de gomme arabique ou de gélatine, la faculté de montée de la crème du lait est améliorée, parce que les dits produits visqueux produisent une agglomération artificielle des globules de matière grasse, de sorte qu'on peut de cette façon régénérer la faculté de montée de la crème du lait pasteurisé. Nous avons confirmé l'essai de RAHN. L'optimum pour la proportion de gomme est de $1 / 2$ à $1 \%$. De plus fortes proportions de gomme entravent à nouveau la montée de la crème, et l'on sait parfaitement qu'on emploie $4 \%$ de gomme pour la stabilisation des émulsions aqueuses de matière grasse.

Ayant ces faits à l'esprit, il est clair que le phénomène en question ne peut pas être expliqué par de petites modifications dans la viscosité du plasma du lait. Comme on l'a vu, l'atténuation dans la faculté de montée de la crème, qui a lieu lors du chauffage du lait, ne peut pas être attribuée à des modifications des membranes dans les globules de matière grasse, mais à une modification du plasma du lait lui-même, et comme la véritable cause est que l'agglutination naturelle des globules de matière grasse cesse peu à ipeu, l'explication peut difficilement être autre que la

(1) PALMER et ANDERSON (Journal of Dairy Science, 1926, pp. 1-13 et pp. 171-191), et WHTTAkmR, Sherman et Sharp (Journal of Dairy Science, 1927, pp. 361.371). 
suivante, c'est-à-dire que le plasma du lait eontient une agglutinine qui agit sur les membranes des globules de matière grasse, et que cette enzyme est tout bonnement détruite par le chauffage du lait. Précisément, les modes de chauffage qui détruisent la montée de la crème du lait détruisent aussi l'agglutinine du sang de bœuf, et comme des investigateurs hollandais ont déjà montré que le sérum du sang de bœuf, et spécialement l'euglobuline qui y est contenue (1) favorise la faculté de montée de la crème du lait, il ne peut y avoir absolument aucun doute à ce que l'agglutinine, ou les agglutinines du lait proviennent du sérum du sang. Comme les agglutinines qui agglutinent les globules rouges du sang ont une température optimum basse, nous avons d'une manière toutà fait spontanós l'explication du phénomène remarquable qui est que la montée de la crème a lieu plus facilement à basse température, quoique la viscosité du plasma du lait soit d'autant plus grande, que la température est plus basse.

Il ne faut pas oublier que, dans cette étude, quand on parle de montée de erème, on ne tient compte que de celle qui a lieu pendant 2 heures dans les conditions indiquées. En 24 heures et même en plus de temps, une partie importante des globules de matière grasse non agglutinés atteignent la couche de crème, de sorte que les différences dans la faculté de montée de la crème, sur lesquelles nous avons basé notre réaction, disparaissent partiellement. Avec le lait de Jersey, cette altération du rapport A pout déjà a voir lieu avant que les deux heures ne se soient écoulées, car les globules de matière grasse de ce lait sont tellement gros qu'ils atteignent rapidement la surface sans être agglomérés ensemble. C'est ce qui explique pourquoi notre réaction de montée de crème ne convient pas si bien pour le lait de Jersey. Par la centrifugation, les globules de matière grasse sont soumis à de si grandes forces que cela ne signifie rien, qu'ils soient agglutinés ou non, et de nombreuses masses de globules gras seront tout de même réduites en morceaux par le traitement mécanique violent, auquel elles sont soumises. Ici, il n'y a que la viscosité du plasma du lait qui semble jouer un rôle, et e'est par la centrifugation qu'on obtient tout de même le meilleur écrémage à des températures qui sont extrêmement nuisibles à la montée naturelle de la crème du lait.

(A suivre.)

\title{
AVANTAGES DES POMPES A ENGRENAGE POUR LA CIRCULATION DU LAIT, DE LA BIËE DES JUS DE FRUITS \\ DANS LES APPAREILS DE PASTEURISATION
}

\author{
par H. STASSANO et A. P. ROLLET.
}

L'un de nous a été amené à adopter le système de la pompe à engre-

(1) VAN DAM, HEKMA, SiRzs et BrouWER (Verslagen Landbouwlound. Onderz. d. Rijks. landbouwproefstation, 1922, 1923, 1924 et 1925). 